\title{
Criminalización del aborto en la ciudad de Córdoba (1887-1922): un estudio de caso
}

\author{
Criminalization of abortion in the city of Córdoba (1887-1922): a case study
}

\author{
Yael Sol Ortiz \\ Universidad Nacional de Córdoba, Facultad de Filosofía y Humanidades, \\ Centro de Investigaciones "Maria Saleme de Burnichon", Argentina \\ yae.ortiz@gmail.com
}

\section{Resumen:}

Mi objetivo es analizar las características y representaciones de las prácticas abortivas en la ciudad de Córdoba a principio del siglo XX, relacionándolas con una incipiente y progresiva criminalización del aborto. En ese sentido, considero que la cuestión del aborto se encuentra inextricablemente relacionada con un proceso a nivel nacional denominado maternalización de las mujeres, que construye a la mujer unívocamente como madre. Atenderé a un recorte temporal anclado en la cultura legal estatal, entre los años 1887 y 1922. Ambos cortes responden, por un lado, a la sancióndel Código Penal y al establecimiento del aborto como "delito contra las personas". Por el otro, a la reforma penal que modifica su tratamiento. Dentro de este lapso he podido registrar, para la ciudad de Córdoba, las primeras causas judiciales caratuladas como "aborto", lo que podría permitirme el acercamiento a la cultura popular. De esta manera, recupero dos campos en tensión en el ejercicio de la justicia: la cultura legal estatal, trazada por agentes estatales, expertos legales criminólogos y comunicadores sociales que sancionan e interpretan las normas legales, establecen procedimientos jurídicos y presentan los peligros que el delito significa a la sociedad; y la cultura popular, que tiene sus propias concepciones sobre esas normas legales. En este artículo me detendré en el análisis minucioso de un expediente judicial sobre la causa abierta a una mujer "por suponérsela autora de aborto violento". Dicho caso permite reflexionar sobre el carácter incipiente y crecientemente condenatorio a dicha práctica y sobre las ambigüedades y limites porosos entre aborto e infanticidio, entre aborto espontáneo y provocado. A su vez, el expediente se presenta como la puerta de acceso a la enunciación de diferentes discursos que ponen en tensión la relación antes aludida. Así, a los planteos expertos (el juez, el fiscal, los médicos, entre otros) se involucran con lxs profanxs (la acusada, lxs vecinxs, etc.).

Palabras Clave: Aborto, Criminalización, Córdoba, Argentina.

\section{AbstraCt:}

My aim is to analyze the characteristics and representations of abortive practices in Córdoba city at the beginning of the 20th century, associating them with an emerging and progressive criminalization of abortion. In that sense, I believe that the abortion matter is inextricably related with a national-level process called women maternalization, which constructs women unequivocally as mothers. I will focus on a particular period which is anchored in the state legal culture and which includes from the year 1887 to the year 1922. My setting of this period's start and end points answers, on the one hand, to the sanctioning of the Penal Code and to the establishment of abortion as a "crime against a person". On the other hand, it answers to the Penal Code reform that modified the treatment of this matter. Within this time span, I could register, in Córdoba city, the first legal cases entitled "abortion", which could allow my approaching of the popular culture. In this way, I have detected two conflicting areas when it comes to the administration of justice: the state's legal culture, drawn up by state officers, criminal legal experts and social communicators who sanction and interpret legal standards, establish legal procedures and present the dangers that criminal actions pose to society; and the popular culture, which has its own conceptions about such legal standards. In this article, I will make a thorough analysis of a judicial file dealing with the open court case of a woman accused of "being the presumptive perpetrator of a violent abortion." Such case allows us to reflect about the emerging and increasingly condemning character of attitudes towards the practice of abortion and about the ambiguities and blurry limits between abortion and infanticide, and between spontaneous and induced abortion. At the same time, the file constitutes an access door for us into the formulation of different discourses that bring tension to the conflictive abovementioned relationships. In this way, expert propositions (the judge, the prosecutor, doctors, among others) become involved with layman ones (the accused woman, neighbors, etc.).

KEYWORDS: Abortion, Criminalization, Córdoba, Argentina. 


\section{INTRODUCCIÓN}

La maternalización de las mujeres fue un proceso que traspasó fronteras nacionales y se desarrolló a lo largo del mundo occidental moderno. En Argentina, entre 1890 y 1940, un conjunto de instituciones y políticas públicas, nutridas por los campos profesionales médico y jurídico establecieron a la maternidad como un "asunto público", promoviendo un mayor control sobre los cuerpos y las prácticas de las mujeres (Biernat y Ramacciotti, 2013; Nari, 2004). En dicho proceso se construyó la idea de que las mujeres tenían como unívoco destino, el de ser madre, estimulando un progresivo solapamiento entre ser mujer y ser madre. La maternidad fue considerada lo natural y "el deber ser" de las mujeres, generando una relación indisoluble, cristalizando un binomio, el de mujer-hijo. De esta manera, los cuerpos de las mujeres solo podían destinarse para la maternidad; otras prácticas como la sexualidad o el trabajo asalariado amenazaban la reproducción, la familia, la sociedad y hasta la "raza". Todas las actividades que no tuvieran una relación directa con la maternidad eran consideradas antinaturales, como podía ser el ocio, el placer, los trabajos e incluso el estudio (Calandria, 2015a; Lobato, 1997; Nari, 2004). En este escenario, la existencia de abortos sugiere prácticas alternativas que tensionan la idea de la maternidad como algo natural e instintivo de las mujeres, poniendo en cuestión aquel binomio naturalizado. El aborto, pero también el infanticidio o el abandono de niños/as mostraron los límites del acatamiento al mandato de maternidad obligatoria.

En este artículo me propongo un primer acercamiento al análisis de la criminalización de aborto y a la reconstrucción de las representaciones y prácticas del aborto en la ciudad de Córdoba entre 1887 y 1922 a partir de un estudio de caso. Para trabajar esta problemática retomo los estudios de la "cuestión criminal" y los estudios de género e historia de/con mujeres.

Los estudios de género y sobre las mujeres realizados en la Argentina han sido abordados historiográficamente desde una perspectiva normativa o desde un enfoque disruptivo (Valobra, 2005). Mi intención aquí es combinar la perspectiva normativa y la disruptiva, observando la relación entre normas y prácticas, pensando dicha relación como variable y no como una imposición de una sobre otra (Calandria, 2015a; De Paz Trueba, 2010). La primera acentúa los roles de las instituciones estatales, los médicos, la Iglesia católica, las sociedades de beneficencia, los discursos "prescriptivos" de diarios, entre otros, en la configuración de las ideas y prácticas genéricas. Dentro de esta línea existen una serie de estudios históricos que acercan a nuestra temática y refieren al proceso de maternalización de las mujeres que se produjo en Argentina (Nari, 2004). En el enfoque disruptivo se tienen en cuenta las voces de las mujeres y su capacidad de agencia. En él se encuentran investigaciones sobre ciertas prácticas que tensionaron la idea de la maternidad como algo natural e instintivo de las mujeres, como son el abandono de niños/as, infanticidios y abortos (Barrancos, 1991; Calandria, 2013, 2014a, 2014b, 2015a; 2015b; Nari, 2004; Piazzi, 2009). Es decir, en este ensayo se aborda la configuración de "normas" de comportamientos y estereotipos a través del accionar de instituciones estatales y de los discursos médicos y jurídicos que propiciaron una idea de mujer unívocamente asociada a su rol de madre. Asimismo, revisaré cómo se dio el proceso de la criminalización del aborto; y, a su vez, recuperaré las propias prácticas de las mujeres -los abortos- que ponen en tensión la idea de la maternidad como el destino "natural" de las mujeres.

Por otro lado, retomo ciertos avances de los estudios de la "cuestión criminal” (Sozzo, 2009) que me permitirían abordar al aborto como "delito", como un constructo social y político. El delito es concebido como un artificio que se plasma, en un primer momento, en la ley penal aunque también se le otorga significado desde lo concreto y particular, a partir de actores estatales y no-estatales, instituciones, discursos y prácticas. En esta línea, recupero la propuesta de Salvatore (2010) sobre dos campos en tensión y relación en el ejercicio de la justicia: la cultura legal estatal, trazada por agentes estatales, expertos legales criminólogos, educadores y comunicadores sociales que sancionan e interpretan las normas legales, establecen procedimientos jurídicos y presentan los peligros que el delito significa a la sociedad; y la cultura popular, que tiene sus propias concepciones sobre esas normas legales, expresando distintos ideales de justicia. Su propuesta 
resulta sugerente para reconstruir una cultura legal estatal sobre el aborto (leyes y discursos profesionales) en diálogo con una cultura popular, una enunciación subalterna pasible de encontrarse en los expedientes judiciales. Siguiendo esto último, atenderé a un recorte temporal anclado en la cultura legal estatal, entre los años 1887 y 1922.

El proceso de modernización y consolidación del Estado implicó un avance en la codificación de sus alcances punitivos a través del Código Penal de 1887 en el que se estableció el aborto como un delito contra las personas, articulado que posteriormente fue modificado y puesto en vigencia en 1922. Dentro de este lapso he podido registrar, para la ciudad de Córdoba, las primeras causas judiciales caratuladas como "aborto", comenzando una tendencia a judicializar casos de abortos y presentando indicios del comienzo de una intolerancia y persecución hacia esta práctica. La penalización estatal y el comienzo en la judicialización de casos de aborto nos presentan indicios del comienzo de la criminalización del aborto. De esta manera, se va conformando una cultura legal estatal sobre el aborto a partir de la legislación vigente y las prácticas judiciales, a la vez, los expedientes judiciales me permitirán un acercamiento a la cultura popular.

Los expedientes judiciales han sido revalorizados como fuentes históricas por los estudios sobre los sectores subalternos para reconstruir la historia de grupos marginados-subordinados y reposicionarlos como sujetos activos y políticos. A su vez, la incorporación de las mujeres en la historiografía trajo como desafío la búsqueda de nuevas fuentes que nos permitieran reconstruir su historia o la relectura de fuentes tradicionales desde una nueva lupa. En el caso de mujeres de los sectores subalternos, esta búsqueda es a contrapelo, porque las mismas no participan de asociaciones civiles, no escribían diarios personales o cartas ni fueron objeto de biografías. Pero el ámbito judicial presenta un archivo donde sus voces pueden ser encontradas y fragmentos de su vida reconstruidos. Sin embargo, estos expedientes son registrados por la mano de un varón, el juez u otro funcionario judicial. De esta manera, se trata de la producción por varones atravesados por intereses y tensiones de género y también de clase. A su vez, los expedientes judiciales son parte del engranaje estatal y no ofrecen un testimonio directo o un simple reflejo de la experiencia de los/as sujetos/as subalternos porque se encuentran siempre mediados por relaciones de poder en la que se inscriben dichos documentos (Gallucci, 2010). Teniendo en cuenta dicho contexto de producción de las fuentes judiciales a la hora del proceso de investigación, los expedientes me permitirán acceder a fragmentos de enunciación subalterna (Salvatore, 2010) de las otras mujeres, aquellas que no pertenecían a los sectores dominantes, y que en estas causas fueron acusadas de abortar o de practicar abortos.

En este artículo me detendré en el análisis minucioso de un expediente judicial sobre la causa abierta a una mujer "por suponérsela autora de aborto violento". Dicho caso permite reflexionar sobre el carácter incipiente y crecientemente condenatorio a dicha práctica y sobre las ambigüedades y limites porosos entre aborto e infanticidio, entre aborto espontáneo y provocado. A su vez, el expediente se presenta como la puerta de acceso a la enunciación de diferentes discursos que ponen en tensión la relación antes aludida. Así, a los planteos expertos (el juez, el fiscal, los médicos, entre otros) se involucran con lxs profanxs (la acusada, los vecinos, etc.). ${ }^{1}$

$\mathrm{Al}$ mencionado expediente lo selecciono de un corpus documental más amplio, que consistente en un total de 13 causas judiciales caratuladas como "aborto" registradas entre 1887-1922 en los índices del Juzgado Criminal de Córdoba que se encuentra en el Archivo Histórico de la Provincia de Córdoba. Dentro de ese corpus documental se puede identificar un periodo con mayor cantidad de causas iniciadas, entre 1905 y 1913 con 8 casos. Posteriormente, entre 1916 y 1922 hay solo 4 casos, aunque dos de ellos son significativos por tratarse de casos en que una mujer fallece por practicarse un aborto. Las causas judiciales iniciadas se pueden tipificar, en términos generales, en tres tipos de situaciones: mujeres acusadas de abortar; hombres acusados de provocar el aborto de una mujer embarazada; investigación por la muerte de una mujer que abortó,en los cuales se acusa a otra/s mujer/es de haberle realizado el aborto. A su vez, se puede observar que la distinción prevaleciente en las causas judiciales es entre aborto provocado y espontáneo, dentro del provocado no solo están los abortos voluntarios, es decir cuando una mujer decide practicárselo, sino también 
aquello no voluntarios, por ejemplo, en el caso de varones acusados de provocar un aborto a partir del ejercicio de la violencia sobre una mujer embarazada.

Este corpus documental presenta sus complejidades para abordarlo y desmenuzarlo, por lo cual priorizo un estudio pormenorizado de cada una de las causas judiciales. En ese sentido, para un primer acercamiento a mi objeto de estudio decidí centrarme en el período con más casos iniciados por presuntos abortos y, en particular, en un caso que presenta ciertos indicios de tratarse de un aborto voluntario aunque se resuelve como un aborto espontáneo. La riqueza de ese expediente consiste en las relaciones sociales y genéricas que se pueden reconstruir en un ámbito cotidiano de principios del siglo XX. En este caso, el barrio Pueblo General Paz de Córdoba en 1908, donde tres vecinos inician la causa contra Cándida y sus vecinas tienden lazos de solidaridad hacia ella en el momento de encontrarse enferma y cuando realizan sus declaraciones. Destaco, a su vez, las particularidades de la protagonista del expediente, una mujer joven que vivía sola y trabajaba en una fábrica radicada cerca de su hogar, por lo cual, presenta el perfil de una mujer sin autoridad masculina a su alrededor y que solventaba por sí misma su supervivencia en los inicios del siglo XX.

\section{LA CRIMINALIZACIÓN DEL ABORTO: LA LEY Y EL JUZGADO}

Se puede encontrar una primera referencia legal al aborto en Fuero Juzgo (Libro VI Titulo IV Ley VII), que era parte de la doctrina jurídica de origen español utilizada durante el periodo colonial. En ella se condenaba a la pena de muerte o la ceguera a la mujer por "prender yerbas por abortar", a su vez, tanto el aborto como el infanticidio recibían la misma condena (Piazzi, 2009; Vasallo, 2012).

Carlos Tejedor ${ }^{2}$ en su Curso de derecho criminal planteaba que "el aborto acaba en efecto donde empieza el infanticidio, y es un delito menor que este, porque la mujer embarazada no es madre todavía". (Tejedor, 1860: 228) Esta es la definición que podemos encontrar de aborto en nuestro periodo de estudio, con límites difusos entre infanticidio y aborto, tanto en su definición como en el momento de comprobar el delito (Piazzi, 2009).

En la Argentina de principios del siglo XX el aborto es tipificado como un delito contra las personas, de acuerdo con el Código Penal (CP) sancionado en 1887. Se incluye que si el motivo del aborto fuera ocultar la deshonra de la mujer se debería aplicar el mínimo de esta pena, cuestión que no se menciona en el CP sancionado en 1921. Sin embargo, no se establece una definición de lo considerado aborto, solamente se tipifican las condenas y se presentan dos potenciales acusados/as: la mujer que decidiera abortar y quien le realiza el aborto, pero si este/a último/a es un profesional de la medicina le correspondería un castigo mayor, siendo inhabilitados/as por un tiempo estipulado. Se puede considerar a dicho articulado como un intento de disciplinar a estos/as profesionales que facilitaban el acceso a un aborto a mujeres o familias que así lo decidieran. En ese sentido, la sospecha de que las parteras realizaban abortos va a ser una constante en el discurso médico, higienista y jurídico.

La cristalización del aborto como un delito en el CP del recientemente consolidado Estado Nacional Argentino marca un inicio en la criminalización de esta práctica y de las/os sujetos que participaban del mismo. Sin embargo, no implicó su inmediata persecución. En el último tercio del siglo XIX en el Juzgado Criminal de Córdoba, encuentro solamente dos causas judiciales caratuladas como aborto, una en 1873 y otra en 1884. Pero en las primeras décadas del siglo XX aumenta su cantidad. Entre 1901 y 1922 hubo 18 causas judiciales en el Juzgado Criminal de Córdoba caratuladas como posibles abortos, 13 ocurrieron en la ciudad de Córdoba y 5 en los pueblos de los alrededores de la misma.

En cambio, los casos de infanticidios judicializados fueron más numerosos, en el último tercio del siglo XIX fueron registrados 88 casos (Candia y Tita, 2005). El infanticidio fue un delito recurrente en la sociedad cordobesa de fines del siglo XIX y principios del siglo XX, considerado el crimen contra la maternidad por antonomasia, en comparación el aborto parece haber sido una preocupación social más marginal.

El aumento de causas judiciales no implica necesariamente un aumento en la cantidad de abortos, sino un aumento en su denuncia y judicialización. En estas causas jueces y fiscales se encuentran con la dificultad 
y casi imposibilidad de comprobar fehacientemente la existencia de un aborto provocado, ni siquiera los médicos en los informes que les solicitan desde el Juzgado pueden dar un respuesta firme. Como planteó un abogado en una causa judicial: "la circunstancia de que un aborto por intervención de medios mecánicos no debe dejar rastros, cuando es la obra de una mano experta”. De esta manera, el aborto fue incluso más difícil de comprobar que el infanticidio. De esa dificultad, se deriva que los expedientes judiciales concluyan sobreseyendo a las/os acusadas/os, y, de esta manera, no hay pruebas concluyentes de que el aborto se haya realizado. La excepción son los casos en que se investiga una muerte a causa de aborto, donde la existencia del mismo es demostrada sin ambigüedades. Ante las limitaciones para comprobar un aborto provocado en el cuerpo de la mujer, cobró centralidad el feto como prueba legal, prueba que en muchos casos faltó. Con los análisis médicos al feto buscaban determinar, por un lado, si fue un aborto o un infanticidio y, por otro, si fue un aborto provocado o espontáneo.

\section{El CASO de CÁNDida Leones: "SUPUEsta AUTORa de aborto Violento"}

En 1908, Cándida Leones fue acusada de cometer un "aborto violento", primero por tres vecinos a la policía y, posteriormente, en una causa judicial, ${ }^{3}$ por la cual estuvo detenida en el Asilo del "Buen Pastor" durante dos meses. Sin embargo, no se pudo comprobar que el aborto haya sido provocado y fue sobreseída. Comparte esta característica con otros casos de acusaciones a mujeres embarazas por abortar: la imposibilidad de comprobar la realización del mismo o su carácter de provocado.

\section{LOS VECINOS: MECANISMOS DE CONTROL}

Tres vecinos le señalan al cabo 2do Francisco Moyano que Cándida se encontraba enferma en su domicilio, consideraban que estaba embarazada desde hacía un tiempo y sospechaban que la causa de su enfermedad era el parto y que Cándida "había cometido el delito de infanticidio dado el misterio que guardaba con relación á su enfermedad" (Folio 1). A su vez, planteaban que no era "el primer crimen de la León, de ésta índole". Fueron ellos quienes se sintieron interpelados por sus sospechas y lo plantearon ante la Policía.

La trasmisión de información de manera informal fue el puntapié inicial para que esta causa judicial existiera, con una acusación doble: estaba cometiendo un crimen (infanticidio) y ya había cometido otros anteriormente. Se puede pensar el accionar de estos vecinos como parte de la cultura popular sobre la penalidad, pero en este caso no como un rechazo a las normas legales sino colaborando y promoviendo una investigación judicial.

Dos de estos varones volverán a declarar una vez avanzado el caso: Lucrecio Murua ( 52 años, jornalero y casado) y Filemon Freites (52 años, viudo y comerciante). Los vecinos pueden precisar que Cándida tenía signos de estar embarazada, uno de ellos consideraba que era el tiempo en que correspondería para que pariera y el otro puntualizaba que durante ocho días no fue al trabajo. Estas declaraciones señalan ciertos mecanismos de control por parte de los vecinos sobre el comportamiento de Cándida. Freites es comerciante y es quien detalla que no la vio pasar por ocho días, es decir tenía un seguimiento de su recorrido diario, posiblemente por su actividad de comerciante. La figura del rumor entre los vecinos asume un lugar central, conformando la acusación: "estas habladurías son cosas que suponen los vecinos, pero tal vez no ha ocurrido" (1908, folio 21). Rumor del que estos mismos varones podrían haber sido participes en su creación; y lo fueron, en particular, en su difusión para que llegara hasta oídos del cabo segundo Francisco Moyano. 


\section{LAS VECINAS: MECANISMOS DE SOLIDARIDAD}

$\mathrm{Al}$ comienzo del procedimiento judicial el Juez Lavisse ordena la prisión preventiva para tres mujeres consideradas sospechosas de complicidad. Las mismas también eran vecinas de Cándida y fueron alojadas en el Asilo del Buen Pastor. Estas vecinas eran: Tomasa Lujan (de 40 años, viuda y dedicada a los "quehaceres domésticos”), Facunda Pereyra (50 años, soltera y lavandera) e Inocencia de Arrascaeta (35 años, casada y costurera).

Al preguntarles si sabían si la acusada sufría de una "enfermedad repentina últimamente” y de qué tipo era; si le había comentado o notaron que estaba embarazada; si sabían que hubiese abortado; si le dieron remedios y de qué tipo eran; cuál era su conducta y carácter; y si sabían que haya estado embarazada o realizado un aborto previamente, las vecinas respondieron que la conocen hace poco tiempo y que no poseen sospechas sobre sus posibles embarazos.

Las preguntas orientadas al suministro de medicamentos ¿podría estar introduciendo la sospecha de que la acusada haya tomado algún remedio de tipo abortivo? Por el momento, no hay indicios claros de que esos remedios hayan podido ser abortivos. Los remedios e infusiones que utilizó Cándida eran previstos y preparados por ella, ante lo cual se puede suponer cierto conocimiento de hierbas curativas y que las dos mujeres solo colaboraron con lo que Cándida les pedía.

A lo largo de las declaraciones registradas en el expediente, se observan ciertas relaciones cotidianas de Inocencia y Tomasa con Cándida: Inocencia le cocinaba y le preparó ciertas infusiones; y Tomasa le calentaba agua en su casa y luego se la alcanzaba.

A pesar de que muestran distancia afectiva con ella, buscando limitar la posibilidad de quedar ligadas con un delito, las declaraciones de las vecinas no presentan señales de sospecha ni acusación alguna, por el contrario aparecen ciertos mecanismos de solidaridad vinculados a tareas cotidianas y de acompañamiento.

\section{LA ACUSADA, EL JUEZ, LOS MÉDICOS Y EL FISCAL: ¿RESOLUCIÓN DEL CASO?}

Cándida era una mujer de aproximadamente 25 años, soltera y vivía sola; trabajaba en una fábrica de papel, que probablemente quedaba en su mismo barrio. La descripción de sus rasgos en el expediente presenta una mujer de color moreno, ojos negros y facciones regulares. Quienes la visitaron desde el Consejo Provincial de Higiene consideraban que era "sumamente incomodo el domicilio donde se aloja” (F.14), lo cual da la pauta de un hogar humilde. Los mismos vecinos que la acusaron, la describieron como trabajadora, humilde, de buena conducta, carácter tranquilo y con buena relación con sus vecinos.

El expediente no brinda información sobre su familia, salvo la existencia de un hermano, Felimo León, quien no viviría con ella. Es probable que su familia no fuera originaria de Córdoba ni que radicara en dicha ciudad, por lo cual se puede intuir que Cándida era una mujer que se mantenía económicamente. Es de destacar que poseía cierta instrucción y podía firmar con su nombre.

El expediente detalla que al inicio del procedimiento el comisario M. Arguello ingresó al domicilio de Cándida para decidir si daría curso o no a las acusaciones presentadas; al encontrarlas como fundadas convocó y al domicilio el Juez del Crimen de turno, Dr. José Lavisse, y el Dr. Luna. Este último consideró que Cándida había dado a luz, por lo cual la acusación de los vecinos pasa a ser una causa judicial. Ante las preguntas que la Policía, el juez y el médico de la policía le realizan en su domicilio, Cándida primero niega haber estado embarazada, o cometido un infanticidio y "persiste en aseverar que solo fué una hemorragia y que bien pudo ser aborto, pero á pesar de ésta aseveración, no sabe precisar el sitio ó lugar donde ocultó este residuo, encerrándose como digo en frivolas evasivas" (1908, folio 1) (las cursivas son mías). Posteriormente, el juez Lavisse y otro funcionario judicial proceden a tomarle la declaración indagatoria a Cándida, cuando la interrogan si conoce cuál es la causa de su detención, ella asume que porque la consideran "autora de aborto violento" (1908, folio 4). Luego habla de la suspensión de su "enfermedad mensual” (1908, folio 4), 
es decir, su menstruación, y considera que la causa es otra enfermedad, la misma que le habría generado un derrame, el cual "presume sea el aborto" (1908, folio 4). Da una fecha para el aborto, el 12 de mayo, estas declaraciones indican que pudo tratarse de un aborto espontáneo. Ella atribuye el motivo del aborto a unos "sueños horribles" (1908, folio 5) que tuvo la noche anterior y también a su tipo de trabajo, ya que apretaba su vientre contra la mesa en determinados momentos de su labor.

De esta manera, puede decirse que es ella quien introduce el tipo de delito que se incorpora en la caratula del caso, no se siente interpelada por la posibilidad de un infanticidio. Ella cuenta su experiencia del aborto, con fechas y detalles que acompañan su versión; y dado que lo único que los médicos y los testigos pueden aseverar es su embarazo, su declaración tiene peso. Ella cuenta cómo sucedió el aborto:

hacía dos meses que había tenido relaciones amorosas con un individuo, y que el aborto se produjo el doce del mes pasado, más o menos, sin que la declarante hiciese nada para provocarlo, que habiendo (...) a satisfacer una necesidad, (...) la casa no tiene letrina, llego a una barranca que está detrás de su casa en dirección al poste, (...) y al sentarse tuvo un pequeño derrame acompañado de fuertes dolores, entonces la declarante al sentir eso se levantó y como a una cuadra tuvo otro derrame al volverse sentar, creyendo fuera el aborto que enseguida volvió a su casa (...) y se acostó sintiéndose desfallecida, que no obstante esto al otro día concurrió al trabajo (...) de la fábrica de papel, (...) pero como a los tres días después del aborto se le empezaron a hinchar las piernas y pies, y que (...) cayó a la cama y no pudo seguir trabajando (1908, folio 4).

A partir de la versión de Cándida, se podría decir que el aborto fue tres días antes de caer en cama. Luego del mismo se le hincharon las piernas y pies, que fue lo que conversó con algunas vecinas, luego enfermó y faltó durante 8 días al trabajo, periodo en el cual sus vecinas Inocencia y Tomasa la ayudaron a prepararse algunos remedios o infusiones. Y es esta situación la que despierta las sospechas de los vecinos que la denuncian, esta "repentina "enfermedad" y que no la vieron pasar al trabajo por dicho periodo de tiempo.

En su declaración, Cándida plantea que tuvo una "relación marital" que resultó en embarazo. Pero ninguna de las vecinas sabe si mantuvo o no "relaciones amorosas con algún hombre". Por lo cual puede ser una aclaración para salvar las apariencias sobre la situación en que quedó embrazada y no recibir condenas morales por su proceder. A pesar de un embarazo fuera de una pareja estable, nadie la acusa de una vida "indecorosa" ni hay referencias al "deshonor".

Cuando se le pregunta cuánto tiempo estuvo embarazada dice que cree que cuatro meses, porque "ese fue el tiempo que duró la suspensión del flujo mensual” (1908, folio 5). Sin embargo, los médicos no plantean lo mismo, como se verá a continuación. Se la interroga insistentemente por el lugar preciso donde abortó, ante esto ella cuenta que "se asustó mucho, motivo por el cual no recuerda el punto preciso pues casi perdió el conocimiento" (1908, folio 5) y "que antes de regresar a su casa le tapo con arena, que no ha contado a nadie porque tenia miedo y vergüenza de decirlo" (1908, folio 6). Fue con el comisario a hacer un reconocimiento del lugar, pero no pudieron encontrar los restos buscados.

El examen de un médico del Consejo Provincial de Higiene se realizó en el Hospital "San Roque” con el fin de "informar, si en dicha mujer se notan signos demostrativos de preñes pasada, época de embarazo y señales de expulsión violenta del feto, como también las demás circunstancias que se relacionen con unaborto provocado" (1908, folio 16) (las cursivas son mías). Sobre lo cual concluye:

Por los antecedentes expuestos deduzco: $1^{\circ}$ que Cándida León ha tenido familia; $2^{\circ}$ aunque la mayoría de los signos apuntados [comprenden] también á un aborto, es probable que en este caso, dando el estado de su pared abdominal, el parto se haya verificado a término, excepción hecha de que haya habido preñeces anteriores; $3^{\circ}$ la fecha probable del desembarazo á juzgar por el estado de los (...), han sido en los primeros días del mes de Mayo encontrándose en consecuencia preñada desde el mes de Agosto del año anterior; $4^{\circ}$ no hay signo claro de aborto provocado, el que por otra parte no suelen dejar rastro, cuando las maniobras para producirlo, han sido bien practicados (1908, folio 17).

Este examen médico legal plantea un embarazo a término, aunque para plantear la fecha de inicio del embarazo simplemente se calcula que fue 9 meses antes del mes en que se supone que tuvo lugar el parto, sin otro indicio. A su vez confiesa que no pueden comprobar si fue un aborto porque si las maniobras son bien practicadas, no deja rastro. 
Luego de la declaración de las vecinas, el examen médico-legal realizado por el Consejo de Higiene y la nueva declaración de los vecinos denunciantes y el médico de la policía, el juez procedió a ordenar la detención de Cándida, trasladándosela al Asilo del "Buen Pastor".

Posteriormente, el fiscal Rohe solicita el sobreseimiento provisional de la acusada, planteando que los exámenes periciales del Consejo de Higiene y del médico de la policía no son concluyentes. Porque el primero plantea que "no hay signos claros de aborto provocado" (1908, folio 26) y en el segundo porque no pudieron realizar "el examen de la vulva sin el cual no puede adelantarse nada sobre la violencia del aborto" (1908, folio 26). Sólo pueden probar el embarazo por el estado de la pared abdominal y su posibilidad de lactancia. Así pone en contraste dos posiciones: por un lado estos exámenes establecen la posibilidad de un parto a término, pero, por otro lado, Cándidaestima que su embarazo fue de cuatro meses. La comprobación del embarazo podría indicar la existencia del delito, pero debería estar acompañada por otros indicios, en particular "la presencia del feto para constituir prueba legal" (1908, folio 26). De esta manera, considera que Cándida tuvo un aborto espontáneo no provocado. El juez considera lo planteado por el fiscal y procede con el sobreseimiento provisional, porque si bien existen indicios de culpabilidad por parte de Cándida León, se carece de una prueba legal.

\section{Consideraciones finales}

La cultura legal estatal sobre el aborto estuvo conformada por su penalización directa por parte del Estado a través del CP desde 1887, pero, a su vez, por las causas judiciales caratuladas como aborto, que nos dan indicios del comienzo de la persecución de dicha práctica. En la ciudad de Córdoba esa situación comienza a observarse en las primeras dos décadas del siglo XX.

En el expediente se cruzan saberes expertos y profanos. Los discursos expertos de médicos y funcionarios judiciales como el juez y fiscal también aportan a la conformación de una cultura legal del aborto. Los médicos cumplen un rol de importancia al poder brindar pruebas sobre la existencia del delito. En ese sentido, los informes médicos son centrales para la resolución de casos de abortos y para poder distinguir si existió un infanticidio o un aborto. Sin embargo, tienen dificultades para determinar cuál es el tipo de delito practicado. Como se vio, la prueba más importante para poder comprobar la existencia de un aborto provocado es el feto, porque no existirían otros exámenes -realizados en el cuerpo de la mujer- que permitan determinarlo con exactitud. El Informe del Consejo de Higiene plantea que un aborto bien practicado no deja rastro, un punto clave para entender la imposibilidad de establecer una condena. Estas limitaciones demuestran la dificultad que se presentaba para perseguir este "delito".

Por su parte, los funcionarios judiciales son también relevantes, el juez y el fiscal. Es de destacar que no realizan referencias al articulado específico sobre el delito de aborto del CP. Cuando el juez ordena la detención de Cándida es a partir de la sospecha de que realizó un infanticidio, después del Informe del Consejo de Higiene. El fiscal es quien propone su inocencia al considerar que tuvo un aborto espontáneo, y, para ello, confrontó las declaraciones de la acusada y los datos que presentaron los médicos en la causa y, también, aquellos que no pudieron confirmar.

Por otro lado, el expediente presenta otros actores, los/as vecinos. Sus testimonios son muy valiosos porque permiten delinear algunos rasgos de una cultura popular sobre el ejercicio de la justicia en general y sobre el delito del aborto en particular. Cándida era una mujer humilde, trabajadora, soltera y sin una autoridad masculina a su alrededor. Quienes intervienen activamente en esta acusación son tres vecinos varones que buscan que este y otros "crímenes de esta índole" no queden impunes, tomando en sus propias manos la iniciativa de planteárselo a la policía. Aunque presentan ambigüedades al ratificar sus acusaciones y luego aminorarlas diciendo que podían ser solo rumores o habladurías, se puede observar un sesgo condenatorio hacia esta mujer trabajadora, soltera y sin hijos/as. En cambio, se puede observar que sus vecinas tienden lazos 
solidarios al no acusarlas, sino dar buenas referencias y al acompañarla con comidas e infusiones durante su período de reposo.

A pesar de que no se pudo comprobar la existencia de un aborto voluntario y provocado por parte de Cándida, las ambigüedades se mantienen. Por una parte, a través de las acusaciones de los vecinos que descansaban sobre rumores de aborto o infanticidio. Por otra parte, es probable que la sospecha de complicidad o encubrimiento recayera sobre las vecinas, lo cual no es corroborado. A su vez, la preparación de remedios utilizando hierbas demuestra ciertos saberes medicinales por parte de Cándida y la presunción de que haya utilizado estos saberes populares para abortar se puede entrever en las preguntas direccionadas sobre este particular en varios de los interrogatorios, pero que no implicó ninguna acusación directa. Por último, la causa presentada por Cándida es que sus tareas laborales hayan inducido el aborto, siendo así un aborto espontáneo, cuestión que invita a indagar sobre las actividades cotidianas en la fábrica.

Desde principios del siglo XX, los discursos médicos que forman parte del proceso de maternalización de las mujeres y los discursos jurídicos cristalizados en las normas legales y en los expedientes judiciales elevan los umbrales de intolerancia al aborto. En ese sentido, se puede pensar el comienzo de un cambio en las sensibilidades que dará lugar a que sea el aborto el crimen por antonomasia contra la maternidad ya avanzado el siglo XX. Entre 1887 y 1922, el aborto comienza a tomar cierto protagonismo en el debate público, pero todavía con un carácter marginal. Este periodo se diferencia de uno inmediatamente posterior, en la década del treinta y cuarenta, cuando la cuestión del aborto se posiciona claramente en el debate público al relacionarlo con la baja en la tasa de natalidad, es decir con una preocupación poblacional, y consideran al aborto como una causa de la "desnatalidad" de la Argentina.

Este trabajo es una primera aproximación a la criminalización de la práctica abortiva en la ciudad de Córdoba a principios del siglo XX. Se puede especular que los casos que llegaban a la justicia eran excepcionales, en tanto la mayoría se mantenían en el silencio. A su vez, estas causas judiciales terminaron con sobreseimientos al no poder comprobar la realización de un aborto provocado. Igualmente estos casos que trató la justicia permiten concluir que en dicho periodo comienza una tendencia a judicializar casos de posibles abortos provocados, lo cual considero como un indicio del comienzo de una criminalización del aborto.

\section{ReFERENCIAS}

Barrancos, D. (1991). Contracepcionalidad y aborto en la década de 1920: problema privado y cuestión pública. Estudios sociales, 1, 75-86.

Biernat, C. y Ramacciotti, K. (2013). Crecer y multiplicarse: la politica sanitaria materno-infantil argentina 1900-1960. Buenos Aires: Biblos.

Caimari, L. (2007). Ley de los profanos. Buenos Aires: Fondo de Cultura Económica.

Calandria, M. S. (2013). ¿Madre se nace o se hace?: Mujeres infanticidas y discurso jurídico a principios del siglo XX. En Jornadas CING. FAHCE-UNLP, La Plata, Argentina. Recuperado de http://sedici.unlp.edu.ar/handle/10 $915 / 41476$

Calandria, M. S. (2014a). "Entre la honra y la vida": un análisis microhistórico sobre mujeres infanticidas en la provincia de Buenos Aires 1904-1913. e-l@tina Revista electrónica de estudios latinoamericanos, 2(47).

Calandria, M. S. (2014b). Una mirada desde el margen: el caso de la infanticida María Mancilla (Buenos Aires, 1904). Cuadernos de Hideas, 8(8). Recuperado de http://perio.unlp.edu.ar/ojs/index.php/cps/article/view/2345

Calandria, M. S. (2015a). Entre obstáculos y posibilidades metodológicas: género y Estado en un proyecto de investigación. Estudios sociales del estado, 1(2), 155-173.

Calandria, M. S. (2015b). Maternidades en cuestión: modelos idílicos y prácticas de las madres en Argentina 1890-1936. Trabajos y comunicaciones (41). Recuperado de http://www.trabajosycomunicaciones.fahce.unlp.ed u.ar/article/view/TyC2015n41a07 
Candia, M. A. y Tita, F. A. (2004). Crimen y maternidad. Infanticidio en Córdoba, 18050-1905.(Tesis de grado inédita), Escuela de Historia, Facultad de Filosofía y Humanidades, Universidad Nacional de Córdoba. Córdoba

De Paz Trueba, Y. (2010). Mujeres y esfera pública. La campaña bonaerense entre 1880 y 1910. Rosario: Prohistoria Ediciones.

Gallucci, L. (2010). Las fuentes judiciales y el estudio de los sectores subalternos. Desafíos y posibilidades de su relación en la investigación historiográfica. Revista electrónica de fuentes y archivos.

Lobato, M.Z. (1997). El Estado en los años treinta y el avance desigual de los derechos y la ciudadanía. Estudios sociales, $7(12), 41-58$.

Nari, M. (2004). Politicas de maternidad y maternalismo politico: Buenos Aires (1890-1940). Buenos Aires: Biblios.

Piazzi, C. A. (2009). Homicidios de niños: legislación, honor y vínculos entrañables (Rosario, segunda mitad del siglo XIX). En M. Sozzo (comp.), Historias de la cuestión criminal en la Argentina (pp. 165-190). Buenos Aires: Editores del Puerto.

Salvatore, R. (2010). Subalternos, derechos y justicia penal. México: Gedisa.

Sozzo, M. (2009). Historias de la cuestión criminal en Argentina. Buenos Aires: Editores del Puerto.

Valobra, A. M. (2005). Algunas consideraciones acerca de la historia de las mujeres y género en Argentina. Nuevo topo, $1,101-122$.

Vasallo, J. (2012). Sexualidad y derecho: algunas notas sobre la regulación de la sexualidad en la Argentina. En J. M. Morán Faúndes, M. C. Sgró Ruata, y Vaggione, J. M. (edits.), Sexualidades, desigualdades y derechos (pp. 125-156). Córdoba: Ciencia, Derecho y Sociedad.

\section{Notas}

1 Tomo la distinción experto/profano de Caimari (2007).

2 Carlos Tejedor (1817 - 1903) fue un jurista y político renombrado de Argentina, titular de la cátedra de Derecho Penal y Comercial de la Universidad de Buenos Aires y, a su vez, fue quien redactó el proyecto de CP.

3 Archivo Histórico de la Provincia de Córdoba, 1908, Criminal, 2da nominación, Legajo 13, Expediente 9. Las próximas citas hacen referencia a este expediente. 